\title{
Case Series of Placental Mesenchymal Dysplasia with Live Birth
}

\author{
Yanqing Kong ${ }^{1}$, , Yanrui Zhang ${ }^{1}$, Hongping Tang', Xueyu Chen ${ }^{2}$ \\ ${ }^{1}$ Department of Pathology, Shenzhen Maternity and Child Healthcare Hospital, Southern Medical University, Shenzhen, China \\ ${ }^{2}$ Department of Neonatology, Shenzhen Maternity and Child Healthcare Hospital, Southern Medical University, Shenzhen, China
}

\author{
Email address: \\ congphe1@sina.com (Yanqing Kong) \\ ${ }^{*}$ Corresponding author
}

\section{To cite this article:}

Yanqing Kong, Yanrui Zhang, Hongping Tang, Xueyu Chen. Case Series of Placental Mesenchymal Dysplasia with Live Birth. International Journal of Clinical and Experimental Medical Sciences. Special Issue: Pathology. Vol. 6, No. 1, 2020, pp. 1-4.

doi: 10.11648/j.ijcems.20190601.11

Received: June 29, 2019; Accepted: December 23, 2019; Published: January 2, 2020

\begin{abstract}
Objective: Placental mesenchymal dysplasia (PDM) is a rare disease, the cause is unknown, some are called mesenchymal stem hyperplasia, and some are called pseudo-vesicular block or vascular malformation. The aim is to report the clinical and pathologic characteristics of three cases of placental mesenchymal dysplasia (PMD). Method: Pathologic and clinical data were retrieved from the electronic medical records. The data were reviewed and summarized by the same pathologist. Results: Maternal age was respective 36 years, 31 years and 33 years. Cesarean section was performed at 39 weeks, 37 weeks and 38 weeks, respectively. The newborns were born with birth weight of 1790, 2370 and 3150 grams, respectively, with placenta weight of 590, 579 and 510 grams respectively. Placenta for pathological examination was performed macroscopically thick-walled vessels. Microscopic observation showed that the part of the stem villus was saclikely dilated, the blood vessels were thickened in the villi, the blood vessel wall was thickened, the villus interstitial was loose, and the mucus was changed. Part of the villus edema and degeneration, no trophoblastic hyperplasia, the final villus structure is normal. Conclusion: The size of the lesions in the Placental mesenchymal dysplasia and the location of the lesion is closely related to the birth weight of the newborn. Detailed histology is necessary for diagnosis and differential diagnosis.
\end{abstract}

Keywords: Placental Developmental Abnormality, Placental Disease, Vascular Disease, Hydatidiform Mole

\section{Introduction}

Placental mesenchymal dysplasia (PMD) is a relatively rare placental lesion, which is often difficult to identify with vesicular blocks due to the lack of awareness of the disease by clinicians. The present study aims to organize and collect the recent two cases of newborn babies in live births, review the clinical and pathological data, and improve the understanding of the disease by clinical and pathologists through previous literature and clinical-pathological analysis.

\section{Clinical Data and Methods}

The data of patients with PMD in this hospital were reported. First women were 36 years old, pregnant 2 was born 1, and Down's screening was high risk. Pre-pregnancy check: herpes simplex virus $1+2$ antibody $\mathrm{IgG}>200.000 \mathrm{RU} / \mathrm{ml}$, rubella virus antibody $\mathrm{IgG} 101.000 \mathrm{IU} / \mathrm{ml}$; cytomegalovirus antibody IgG $55.400 \mathrm{RU} / \mathrm{ml}$; folic acid $54.31 \mathrm{nmol} / \mathrm{L}$ in the middle and late pregnancy; $\mathrm{IgG}$ resistance B potency 128 titer. In the third trimester of pregnancy, the placental parenchyma was uneven, and there were multiple small anechoic areas scattered in the field, which showed a honeycomb change. The patient was not further examined. At 39 weeks of gestation, she was admitted to the hospital due to vaginal bloody secretions. After admission, the ultrasound showed the dark area behind the placenta, and she was given a miscarriage treatment. Subsequently, the fetal membrane spontaneously ruptured, because the pregnant woman has a history of cesarean section, scar uterus, accompanied by cesarean section. A baby girl was born, and the appearance of the newborn was normal. The birth weight was $1790 \mathrm{~g}$, and the Apgar score was 9 at 1 minute and 9 at 5 minutes. Neonatal 
examination: blood routine: total white blood cell count $25.35 \times 10^{9} / \mathrm{L}$, neutrophil absolute value $17.72 \times 10^{9} / \mathrm{L}$, red blood cell count $4.18 \times 10^{12} / \mathrm{L}$; hemoglobin concentration $118 \mathrm{~g} / \mathrm{L}$, total platelet count $60 \times 10^{9} / \mathrm{L}$; biochemical liver function: total bilirubin $162.5 \mathrm{umol} / \mathrm{L}$, total protein $48.5 \mathrm{~g} / \mathrm{L}$, albumin $23.9 \mathrm{~g} / \mathrm{L}$; C-reactive protein $10.09 \mathrm{mg} / \mathrm{L}$; postpartum placenta routine pathological examination. Clinical diagnosis: scar uterus, intrauterine infection, premature infants, low birth weight infants, neonatal anemia, jaundice, hypoproteinemia. The newborn gave active treatment and the condition improved and was discharged from the hospital a week later.

Placental pathology, General inspection: Placenta hypertrophy with size $18 \mathrm{~cm} \times 16 \mathrm{~cm} \times 4 \mathrm{~cm}$, weight $590 \mathrm{~g}$. The fetal membrane was slightly grayish-yellow, umbilical cord length $14 \mathrm{~cm}$, diameter $0.9 \mathrm{~cm}$ with three umbilical vessels, local blood clots on the placenta of the placenta, see the gray saclike expansion area, similar to thick-walled blister-like structures.

Case 2, pregnant women 31 years old, pregnancy 3 production 1, pre-pregnancy examination: cytomegalovirus IgG antibody $94.0 \mathrm{U} / \mathrm{ml} ; 7$ weeks pregnant nausea, vomiting, and other obvious early pregnancy reaction. Pregnancy registration, regular checkups, thalassemia negative, normal sugar screening, Down's screening, prenatal non-invasive genetic testing suggest low-risk pregnancy. Pregnancy $24+2$ weeks of grade III color ultrasound: intrauterine single live birth, equivalent to 23 weeks and 1 day, fetal head circumference, femur, tibia measurement is located under the M-2SD line, fetal weight is lower than the tenth percentile. Prenatal diagnosis is recommended for chromosome analysis, but the woman refused further evaluation. Subsequent ultrasound showed that the gestational age of the fetus was less than 1 week of menopause. At the age of $33+2$ weeks, the grade III ultrasound: intrauterine single-lived fetus, placenta grade II, fetal head circumference, abdominal circumference, femur length, and tibia length are all located under the M-2SD line, and the fetal weight is lower than the tenth percentile.
Fetal intrauterine growth restriction was considered. The placenta is obviously thickened, the internal echo is uneven, and it is honeycomb-shaped. At 37 weeks of gestation, the patient was admitted to the hospital; the cesarean section was performed, and a baby girl was born. The newborn weight was 2370g. Postpartum placental pathology was conducted. Clinical diagnosis: fetal growth restriction, small-for-gestation age, premature infants, scar uterus.

Pathological examination of placenta Gross examination: broken, $24 \times 18 \times 9 \mathrm{~cm}$, thickness $4-5.5 \mathrm{~cm}$, weight $579 \mathrm{~g}$, umbilical cord length $1 \mathrm{~cm}$, diameter $1.5 \mathrm{~cm}$, three umbilical vessels. The membrane of the membrane is gray, and there are a lot of vesicular structures in the fetal surface and the middle part of the placenta. The other placental villi have no special changes in the naked eye. The maternal surface is rough and the texture is slightly brittle.

Case 3, pregnant women 33 years old, pregnancy 4 production 1, pre-pregnancy examination: early nausea and vomiting and other early pregnancy reactions, lasting for 1 month, no history of exposure to toxic radiation. Pregnancy $4+$ month consciously fetal movement, activity so far, pregnancy during the construction of the book, regular checkup, thalassemia, Down's screening, OGTT low risk, smooth pregnancy, no complaints discomfort, patients without dizziness, double lower limb edema. At 38 weeks of this pregnancy, vaginal fluid discharge 7 hours before, because the scarred uterus, the infant was delivered through cesarean section, a baby girl was born, and the newborn weight was $3150 \mathrm{~g}$. Clinical diagnosis: scar uterus; premature rupture of membranes.

Placental pathological examination: the placenta size is $17 \times 17 \times 3.5 \mathrm{~cm}$, the weight is $510 \mathrm{~g}$, the membrane was slightly grayish-yellow, the damage was not complete, the umbilical cord was $14 \mathrm{~cm}$ long, the diameter was $1.4 \mathrm{~cm}$, the cut surface was separate in three, and the part of the maternal surface near the marginal area was expanded. It has a blister-like structure with a range of $12 \times 7 \mathrm{~cm}$.

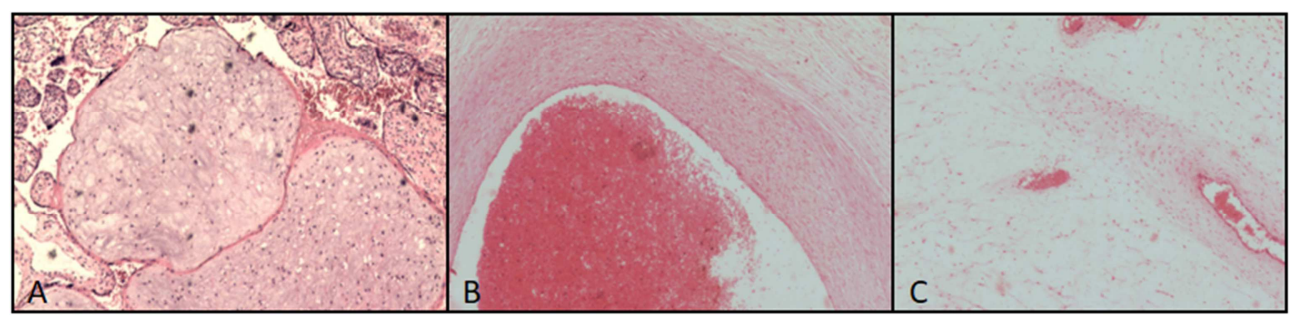

Figure 1. Representative pictures of placental mesenchymal dysplasia (PDM). A, stem villi are saclike, loose connective tissue, normal villi around, no proliferation of trophoblastic cells; $B$, thick-walled vessels with cystic dilatation; $C$, interstitial edema of villi. $100 \times$.

\section{Results}

Case 1 under the microscope: visible lesions of the stem villus saclike expansion, stem villus blood vessels thickened, thickened blood vessel wall, see thick wall small blood vessels, villus interstitial loose, mucinous changes, partial villous edema degeneration, no nourishment leaves The cells proliferated and the terminal villus was normal. Lymphocyte aggregation infiltration was observed in the fetal membrane.
Pathological diagnosis: mesenchymal dysplasia (PMD); stage II of fetal membrane inflammation. Case 2: Microscopic edema of placental villi with mucous-like interstitial, dilated thick-walled blood vessels, partial villi of different sizes, partial villous edema, partial villus interstitial cell proliferation, partial placental villus structure, fetal membrane, and the umbilical cord was slightly edematous. The pathological diagnosis was: dysplasia of the placenta. In the third case, the part of the placenta under the microscope had 
large edema of the villus, accompanied by mucus-like interstitial, and the expanded thick-walled blood vessels were seen individually, and most of the villi were normal. Immune cells infiltrated in the fetal membrane, but not in the umbilical cord. Pathological diagnosis: dysplasia of the placenta.

\section{Discussion}

Placental mesenchymal dysplasia (PDM) is a rare disease, the cause is unknown, some are called mesenchymal stem hyperplasia, and some are called pseudo-vesicular block or vascular malformation. According to the literature, the incidence of PDM is reported at about $0.002 \%$ to $0.02 \%$, or even lower $[1,2]$. The incidence of PDM is difficult to determine because clinical and pathological understanding is inadequate, which may result in overall underestimation and underreporting. On the other hand, it is currently only detected when the placenta is infected or complicated [3, 4]. The incidence of PMD may increase as the pathologist's clinical awareness increases $[5,6]$. At present, the pathogenesis of PMD is still unclear. Chen CE [7] and others believe that the proliferation of stem chorionic mesenchymal cells is accompanied by hyperplasia of mesenchymal cells, and there is a normal villus mesoderm connective tissue in the expanded stem villi. The presence of eosinophilic mucopolysaccharide, presumably congenital mesoderm malformation. Some patients with Beckwith-Wiedemann syndrome are overgrowth syndrome, also known as an umbilical bulge-mega globe-macro-complex syndrome. There are no consistent clinical diagnostic criteria for this disease, which is considered to be the maternal source or parent of the chromosome 11 p15.5 region, which may affect the expression of IGF-2 and $\mathrm{Xp} 22.31$, which is expressed as vascular endothelial growth factor D (VEGF-D) [8]. Deletion of expression of the source imprinted gene [9]. The karyotype of fetal chromosomal examination in PMD patients is normal, and a small number of patients are accompanied by chromosomal abnormalities, including 13-trisomy, triploid and 13q12.11 involving the GJB6 gene [10, 11].

PDM has no clinical special symptoms in early pregnancy. It can be diagnosed by routine prenatal examination or ultrasound. Some of the serum $\beta \mathrm{HCG}$ is normal or slightly elevated, and serum $\alpha$-blood protein is elevated because of PDM surface transformation. The increase in area, as a result of an increased volume of stem villus and increased number of the placenta, is thought to be an increase in alpha-fetoprotein transferred to the maternal circulation [12]. In the third trimester, fetal growth restriction or fetal death may occur, and some amniotic fluid may occur. It may be caused by difficulty in swallowing the fetus with Beckwith-Wiedemann syndrome. In patients with PMD, intrauterine growth restriction during pregnancy, intrauterine stillbirth, premature delivery, severe fetal growth restriction may be due to poor perfusion of the placental stem villus vascular thrombosis and hypoxia or fetal angiogenesis. The complications of the fetus are mainly anemia, thrombocytopenia or microangiopathic hemolysis, which may be related to the abnormal distribution of blood vessels. Its etiology and pathogenesis are unclear and may be related to abnormal development of $\mathrm{X}$ chromosome and trophoblastic cells [13]. The current prevailing view is that the adverse consequences of PMD are mainly concentrated on the fetus, while the influence of PMD on the mother is mainly manifested as eclampsia and pregnancy-induced hypertension. This study found that one of the pregnant women, the lesion in the marginal zone, the fetus was $3150 \mathrm{~g}$, the fetal weight was normal. It suggests that the lesions are small in scope and concentrated in the marginal area of the placenta, with little or no effect on the fetus. Another pregnant woman's lesions were concentrated in the middle of the placenta and the placenta fetal area. The neonatal weight was $2370 \mathrm{~g}$, suggesting that the placental dysplasia may be related to the site of placental lesions. The lesions are concentrated in the center of the placenta, affecting the degree of fetal growth and development.

PMD ultrasonography mainly showed cystic anechoic zone; before 13 weeks of gestation, patients with early ultrasound examination were not obvious, and vesicular lesions could not be detected. As the pregnancy progresses, the congested blood vessels resemble a black-red or grayish-white scorpion-like structure that can be identified in the placental parenchyma, mainly in the place where the chorionic plate is close to the fetal surface, consistent with the placental gross examination. Subsequent ultrasound showed similar similarity to some of the blister-like blocks. The imaging findings of PMD are similar to those of hydatidiform moles, which are easily misdiagnosed before delivery. Placental dysplasia is due to the cystic expansion of some of the villi, which should be differentiated from the vesicular vesicles and the edema of the missed abortion. In this paper, the aneurysm-like dilatation of the placental villus is localized, the local villus is cystic, or accompanied by the formation of mucin-like interstitial and mucus pools, and the terminal villous structure around the other parts is normal, and the nourishing leaves lacking partial vesicular blocks Cell proliferation characteristics [14].

There are few reports on the effect of the virus on the placenta. Rubella virus, cytomegalovirus, and herpes simplex virus are a group of pathogens that cause intrauterine infection in pregnant women. Studies have shown that TORCH infection can inhibit the proliferation of human placental trophoblast cells and arrest human placental trophoblast cells in the G1 phase to induce apoptosis of human placental trophoblast cells, which may be related to the inhibition of Cdc-6 protein expression [15]. The study of folic acid is mainly about the lack of effects on the fetus, as well as the side effects of excessive folic acid intake, which can mask the early symptoms of vitamin 12 deficiency. Vitamin 12 deficiency can cause damage to the nervous system, interfere with antiepileptic effects and induce convulsions, and also affect zinc absorption. There is no relevant research on the influence of the placenta, and further understanding is needed.

In this case, one of the pregnant women was a senior pregnant woman. The literature review reported that 23-42 years old were reported, and there were more reports in 20-30 years old. It is currently considered that there is no significant 
age-related relationship between PDM and age. There was no report on the number of births and the number of maternal births. There was no correlation between the study and the number of maternal births. There may be fewer cases and further research is needed.

Placental mesenchymal placental size of the placental lesions may be an important factor affecting the fetus or infant. However, its pathogenesis and biological basis need further study. Detailed histology is essential for diagnosis and differential diagnosis. Although PMD often exhibits perinatal adverse outcomes, it may be related to the extent of placental dysplasia. With the advancement of ultrasound technology, prenatal identification of these cases is very important to reduce fetal morbidity and mortality. The prognosis is better than a partial hydatidiform mole. Once the PMD is suspected, the fetal karyotype is normal. Through prenatal screening and patient counseling, strict B-ultrasound detection of fetal growth and development, continued pregnancy is acceptable, thus avoiding unnecessary abortion.

\section{Conclusion}

The size of the lesions in the Placental mesenchymal dysplasia and the location of the lesion is closely related to the birth weight of the newborn. Detailed histology is necessary for diagnosis and differential diagnosis.

\section{References}

[1] Tortoledo M, Galindo A, Ibarrola C. placental mesenchymal dysplasia associated with hepatic mesenchymal hamartoma and pulmonary hamartoma [J]. fetal pediatric pathology, 2010, 29 (4): 261-270.

[2] Arizawa M. Nakayama M. Suspected involvement of the X chromosome in placental mesenchymal dysplasia [J]. Congenital Anomalies, 2002, 42 (4): 309-317.

[3] Nayeri UA, West AB, Grossetta Nardini HK, Copel JA, Sfakianaki AK. Systematic review of sonographic findings of placental mesenchymal dysplasia and subsequent pregnancy outcome. Ultrasound Obstet Gynecol. 2013; 41: 366-74.

[4] Gibson BR, Muir-Padilla J, Champeaux A, Suarez ES.
Mesenchymal dysplasia of the placenta. Placenta. 2004; 25: 671-2.

[5] Havva Serap Toru, Esra cobankent Aytekin, Cem Yasar Sanhal et al. We can Diagnose it if we Consider it. Diagnostic Pitfall for Placenta: Placental Mesenchymal Dysplasia [J]. Turkish J Path, 2015, 01310, 1-5.

[6] Subrata P, Kingshuk B, Prabhat C M, et al. Placental Mesenchymal Dysplasia With Normal Fetus: A Rare Case Report [J]. Iran J Pathol. 2017; 12 (3): 307-310.

[7] Chen CE, Chern SR, Wang TY, et al. Pregnancy with concomitant choragioma and placental vascular malformation with mesenchymal hyperplasia [J]. Hum Reprot, 1997, 12 (11), 2553-2556.

[8] Takahashi H, Matsubara S, Kuwata $T$, et al. Changes in expression of vascular endothelial growth factor D-related genes in placental mesenchymal dysplasia [J]. J Obstet Gynaecol Res. 2014, 40 (4): 1145-9.

[9] Ping AJ, Reeve AE, Law DJ, et al. Genetic linkage of Beckwith-Wiedemann syndrome to $11 \mathrm{p} 15$ [J]. Am J Hum Genet, 1989, 44 (5): 720-723.

[10] Cohen M, Roper E, Sebire NJ, et al. Placental mesenchymal dysplasia associated with fetal aneuploidy [J]. Prenat Diag, 2005, 25 (3): 187-192.

[11] Bogdan D, Sabina N V, Gabriela S, et al. A case report of placental mesenchymal dysplasia [J]. Medicine (2019) 98: 8 (e14554).

[12] Parveen Z, Tongson-Ignacio JE, Fraser CR, et al. Placental mesenchymal dysplasia [J]. Arch Pathol Lab Med, 2007, 131 (1): 131-137.

[13] Pham T, Steele J, Stayboldt C, et al. Placental mesenchymal dysplasia is associated with high rates of intrauterine growth restriction and fetal demise a Report of 11 new cases and a review of the literature. [J]. Am J Clin Pathol, 2006, 126 (1): 67-78.

[14] Moscoso G, Jauniaux E, Hustin J. Placental vascular anomaly with diffuse mesenchymal stem villous hyperplasia: A new clinicopathological entity [J], Pathol Res Pract, 1991, 187 (23), 324-328.

[15] Cai Wang, Wang Haili. TORCH effect of infection on human placental trophoblast and its mechanism [J]. M C Heal care C, 2016, 10 (31): 2180-2183. 